\title{
Transient behaviour of deposition of liquid metal droplets on a solid substrate
}

\author{
J. Chapuis ${ }^{1}$ E. Romero ${ }^{2}$ F. Soulié ${ }^{2}$ C. Bordreuil ${ }^{2} \cdot$ G. Fras $^{2}$
}

\begin{abstract}
This paper investigates the mechanisms that contribute to the spreading of liquid metal macro-drop deposited during Stationary Pulsed Gas Metal Arc Welding on an initially cold solid workpiece. Surface tension and inertial effects take an important part in the behaviour of the liquid metal macro-drop, but in this configuration the influence of energetic effects can also be significant. The experimental results are discussed in the light of dimensional analysis in order to appreciate the influence of the process parameters and the physical mechanisms involved on the spreading of a macro-drop. A law is established to model forced non-isothermal spreading.
\end{abstract}

\section{List of symbols}

$c_{p} \quad$ Specific heat $\left(\mathrm{J} \mathrm{kg}^{-1} \mathrm{~K}^{-1}\right)$

$d_{w} \quad$ Wire diameter $(\mathrm{m})$

$D \quad$ Macro-drop diameter (m)

$f \quad$ Frequency $(\mathrm{Hz})$

$g \quad$ Gravity $\left(\mathrm{m} \mathrm{s}^{-2}\right)$

$h \quad$ Height of the macro-drop (m)

I Welding current (A)

$L \quad$ Enthalpy of melting $\left(\mathrm{J} \mathrm{kg}^{-1}\right)$

$L_{d} \quad$ Characteristic length of the droplet (m)

$P \quad$ Welding power (W)

F. Soulié

fabien.soulie@um2.fr

J. Chapuis

julien.chapuis@areva.com

1 AREVA NP CS 40001 - Saint-Marcel, 71328 Châlon sur Saône Cedex, France

2 Laboratoire de Mécanique et Génie Civil (LMGC), Université de Montpellier, CNRS, Montpellier, France
$R \quad$ Base radius of the macro-drop (m)

$r \quad$ Radius of the droplet $(\mathrm{m})$

$t$ Time (s)

$T_{0} \quad$ Initial temperature of the workpiece (K)

$T_{d} \quad$ Temperature of the droplet $(\mathrm{K})$

$T_{f} \quad$ Fusion temperature (K)

$T_{m} \quad$ Temperature of the macro-drop (K)

$u_{d} \quad$ Droplet velocity $\left(\mathrm{m} \mathrm{s}^{-1}\right)$

$u \quad$ Velocity of the contact line $\left(\mathrm{m} \mathrm{s}^{-1}\right)$

$U \quad$ Voltage $(\mathrm{V})$

$V \quad$ Volume of the macro-drop $\left(\mathrm{m}^{3}\right)$

$v_{d} \quad$ Metal input by time unit $\left(\mathrm{m}^{3} \mathrm{~s}^{-1}\right)$

$V_{w} \quad$ Wire feed speed $\left(\mathrm{m} \mathrm{s}^{-1}\right)$

Bo Bond number $\left(\rho g R^{2} \gamma^{-1}\right)$

$\mathrm{Ca}$ Capillary number $\left(\mu u \gamma^{-1}\right)$

$P e \quad$ Peclet number $\left(\rho c_{p} R u \gamma^{-1}\right)$

Pr Prandtl number $\left(\mu c_{p} \lambda^{-1}\right)$

Re Reynolds number $\left(\rho u D \mu^{-1}\right)$

We Weber number $\left(\rho u_{d}^{2} R \gamma^{-1}\right)$

$Z \quad$ Ohnesorge number $\left(\mu \rho^{-0.5} \gamma^{-0.5} R^{-0.5}\right)$

$\gamma \quad$ Surface tension $\left(\mathrm{N} \mathrm{m}^{-1}\right)$

$\mu \quad$ Viscosity $\left(\mathrm{kg} \mathrm{m}^{-1} \mathrm{~s}^{-1}\right)$

$\rho \quad$ Density $\left(\mathrm{kg} \mathrm{m}^{-3}\right)$

$\lambda \quad$ Thermal conductivity $\left(\mathrm{W} \mathrm{m}^{-1} \mathrm{~K}^{-1}\right)$

$\theta \quad$ Contact angle (rad)

\section{Introduction}

The weld bead geometry influences the mechanical integrity and the stability of the process during multi-passes welding in narrow gap process. The weld bead geometry in this complex configuration is governed by the behaviour of fluid phase and by the behaviour of the gas-liquid-solid line. 


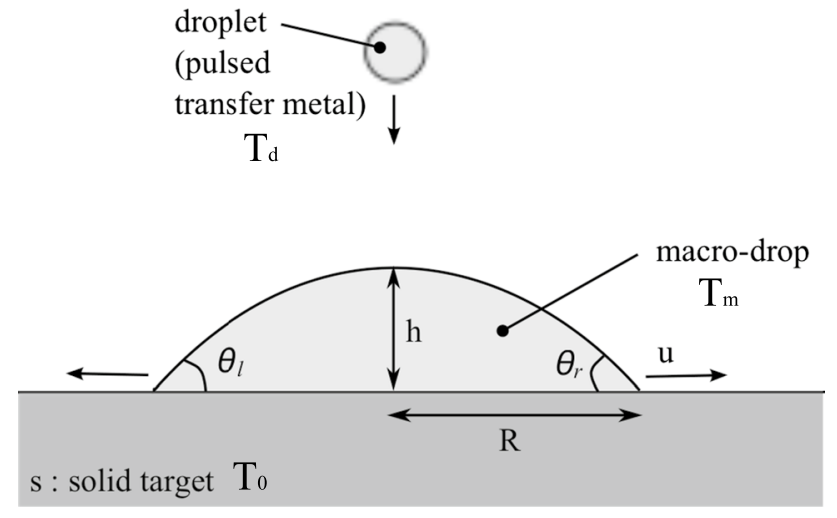

Fig. 1 Deposition and spreading of macro-drop during static GMAW operation. The geometry of the macro-drop is defined by the height $h$, the base radius $R$ defining the base length and the contact angles $\theta$ at the solid-liquid-gas interface (contact line). The speed of spreading $u$ corresponds to the contact line speed

Liquid droplet-solid surface interactions are a subject of interest for scientific research [1-4]. In the case of isothermal configurations, many physical phenomena are involved in the deposition and spreading process, including microscopic and macroscopic phenomena [5, 6]. Complexity increases in non-isothermal configurations in which the influence of solidification and fusion phenomenon appears. In order to study contact-line behaviour, Schiaffino and Sonin [7] developed an experimental test with forced spreading for wax. The forced spreading leads to a macrodrop. It was shown that process parameters influence the contact line motion. In this configuration, m any physical phenomena are related to the spreading of the macro-drop and the contact-line motion is still not well understood. Schiaffino and Sonin [7] worked with small droplets with low temperature $\left(<100{ }^{\circ} \mathrm{C}\right)$. Microdrops with a radius of $25 \mu \mathrm{m}$ and a temperature $T_{0}$ were ejected at constant frequency $f$ and delivered to a target of temperature $T_{0}<T_{f}$. Schiaffino and Sonin [7] demonstrated experimentally that contact line arrest depends not only on material properties but also on thermal conditions.

Similar research works can also be found in high temperature process, such as Pulsed Gas Metal Arc Welding (P-GMAW) where a centimeter metal liquid pool is supplied by a millimeter liquid metal droplet (Fig. 1) [8]. It was shown that the gas-liquid-solid line motion was modified with different process parameters and shielding gas. One of the conclusions was that the base radius of the macro-drop increases like a square root of time (Fig. 2), in agreement with the inertial spreading of liquid drops [9].

Surface tension and inertial effect clearly play an important role in the spreading of the macro-drop, but in this process, thermal effects are significant. The pur-pose of the paper is to investigate the wetting of liquid

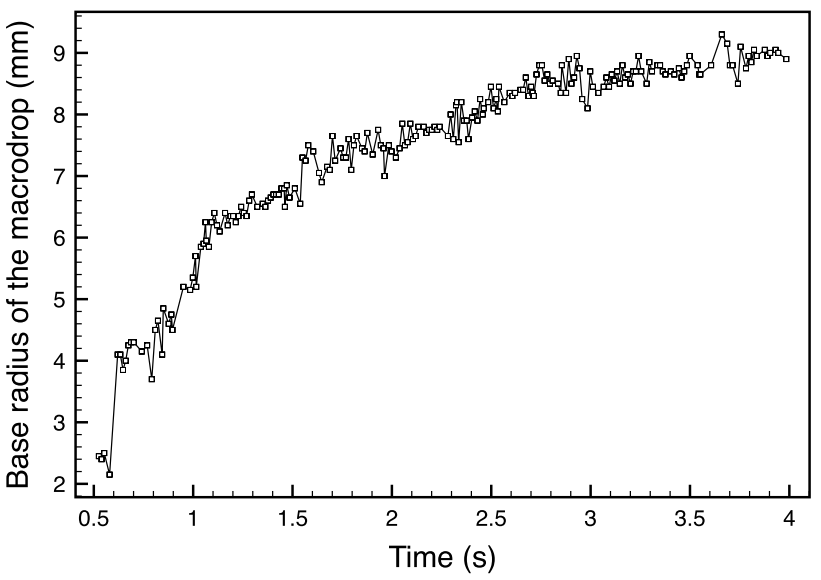

Fig. 2 Evolution of the base radius of the macro-drop with time [8]. In first time $(t<0,5 \mathrm{~s})$, the growth of radius is fast. Then, more the radius is important more the growth is low

metal in this complex application on the base of dimensional analysis and to propose a law of spreading to help engineer to appreciate wetting conditions during welding. The law has to take into account process parameters. Local phenomena around the contact line will be neglected.

To investigate these behaviours, a macro-drop evolution on a flat surface is analysed experimentally. Despite the simpler configuration than narrow gap process, it embeds all physical phenomena.

In this study, dimensional analysis proposed in literature for high temperature process [10-12] is adjusted to our experimental tests performed earlier [8] on the spreading of molten metal.

After a short description of the physical phenomena involved in the macro-drop formation (Sect. 2), the experimental test and the procedure for formulating dimensionless parameters are presented (Sect. 3). The results are discussed in relation with welding and physical parameters (Sect. 4).

\section{Macro-drop formation in Stationary P-GMAW}

The pulsed gas metal arc welding is a common welding process in which electric arc is the main heat source that melts both base material and a continuously-fed metal filler wire to develop a weld pool. A pulsed current waveform (frequency $f$ around $100 \mathrm{~Hz}$ ) is generated between the wire and the weld pool with a peak current around $350 \mathrm{~A}$ and a background current around $80 \mathrm{~A}$. Figure 3 shows the overall configuration of the feeding.

The process parameters are the wire speed $V_{w}$, the wire diameter $d_{w}$, the shielding gas and the material. Depending 


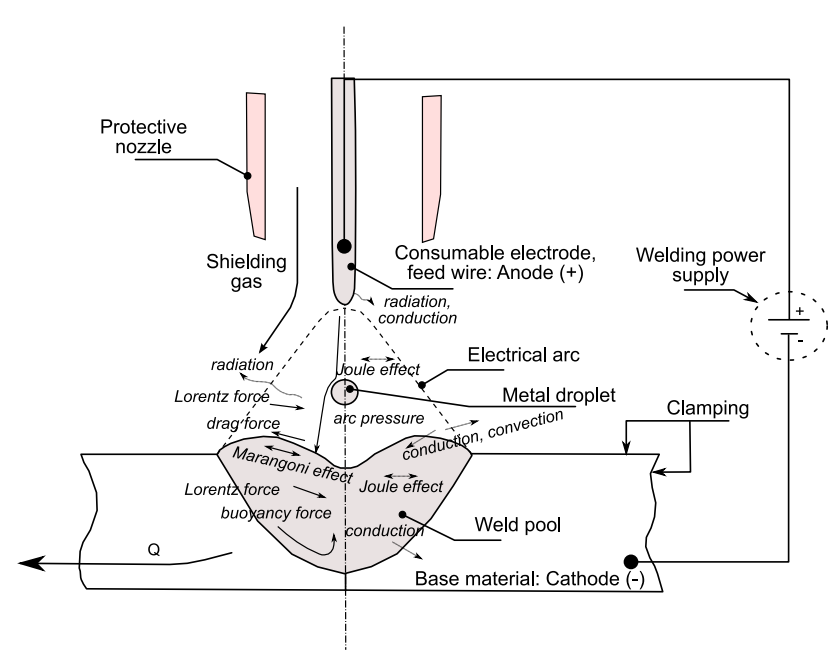

Fig. 3 Gas Metal Arc Welding during static operation; physical description of transfer and weld pool. Droplets are created from the wire and then transfer to the weld pool

on $d_{w}$ and $V_{w}$, arc voltage $U$ and current $I$ are regulated by the generator to melt the wire.

The filler material is melt during the peak current and leads to one droplet per pulse. These droplets have a characteristic size $L_{d}$ (around $1 \mathrm{~mm}$ ), a temperature $T_{d}$ and a velocity $u_{d}$. These values depend on phenomena occurring during breakup of the fluid phase and depend on process parameters. The study of breakup is out of the scope of the present analysis but these values are estimated experimentally just after breakup. In these conditions, the deposition process with arc welding is close to the one analysed by Schiaffino and Sonin [7]. In the present study, the liquid metal deposition consists in a monodisperse spray of droplets of radius $r=0.5 \mathrm{~mm}$. During the free flight through the plasma, some oscillations of the shape of the droplets occur, but droplets follow linear trajectories (approximatively $15 \mathrm{~mm}$ ) from their breakup at the feed wire to their impingement in the weld pool.

The droplets impinge on the weld pool surface at an average speed measured around $0.5 \mathrm{~m} / \mathrm{s}$. This leads to local disturbance in the vicinity of the impact and small amplitude wave propagation on the macro-drop surface. During the peak current, large Lorentz forces are induced into the weld pool and into the plasma. These Lorentz forces increase the pressure in plasma and the surface of the weld pool is deformed due to this high pressure. The weld pool surface behaviour is also driven by the surface tension and gradient of surface tension along the interface between the liquid metal and the gas.

The droplets impinge on a macro-drop of radius $R$ at a temperature $T_{m}$. The target is large (160 mm diameter) and thick $(10 \mathrm{~mm})$ and is homogeneous to the filler material. The droplet were ballistically delivered to the center of the target. The macro-drop has interfaces with the shielding gas and with target.

High speed imaging of static P-GMAW revealed that the macro-drop evolution is driven by heat and mass transfer due to the arc plasma and the deposited droplets. On Fig. 2, the evolution of the base radius (position of the contact line) of the macro-drop was scanned thanks to image detection of the fluid phase [13]. The first rapid advance of the contact line (in the first $1 \mathrm{~s}$ ) is mainly due to the creation of the weld pool due to the arc. In a second phase, the bead continuously grows in volume and spreads over the solid substrate thanks to heat and mass due to the droplets. Figure 2 shows smooth behaviour and some oscillations come from the dynamic motion of the free surface induced by different perturbations (arc and droplets). Studying the geometry evolution can give information on the main local and global phenomena (mass, forces and energy) that influence spreading. In the next part, radius evolution based on previous experimental tests will be analysed through dimensional analysis. The purpose is to see if all the tests with different process parameters can be gathered.

Macro-drop shape is also driven by heat transfer in the liquid. Energy is supplied to the weld pool by the plasma along the weld pool surface and by the heat in the droplet impinging on the weld pool. Energy is taken from the weld pool by heat sink of the solid substrate.

The multi-physics aspect (fluid mechanics and energy conservation) increases the number of parameters that can influence the wetting and a dimensional analysis will help to find the main physical phenomena.

Due to complex coupling of physical equations of the problem and boundary conditions, dynamic and thermal similarity must be derived from dimensional analysis rather than non dimensionalization of the governing equations and boundary conditions. A dimensional analysis can be an interesting way to study their influences [10-12] especially to investigate experimental datas.

\section{Experimental setup and dimensionless procedure}

\subsection{Experimental setup}

In [8], different tests were carried out to see the influence of process parameters on spreading. Experimental setup is briefly reminded here. Stationary spot welds are made using P-GMAW to observe non-isothermal spreading of weld pools with a high-speed camera (4000 fps). Welding current and arc voltage are recorded at $30 \mathrm{kHz}$ sampling rate. The physical characteristics of the material used in the experiments are given in Table 1 (the characteristics of the base metal of the workpiece and of the ER70S steel 
Table 1 Numerical values of physical characteristics [4]

\begin{tabular}{lll}
\hline & Properties (units) & Values \\
\hline$\gamma$ & surface tension $\left(\mathrm{N} \mathrm{m}^{-1}\right)$ & 1.67 \\
$\rho$ & density $\left(\mathrm{kg} \mathrm{m}^{-3}\right)$ & 7200 \\
$\rho_{s}$ & density of the solid $\left(\mathrm{kg} \mathrm{m}^{-3}\right)$ & 7800 \\
$\mu$ & viscosity $\left(\mathrm{kg} \mathrm{m}^{-1} \mathrm{~s}^{-1}\right)$ & 0.006 \\
$c_{p}$ & specific heat $\left(\mathrm{J} \mathrm{kg}^{-1} \mathrm{~K}^{-1}\right)$ & 753 \\
$c_{p s}$ & solid specific heat $\left(\mathrm{J} \mathrm{kg}^{-1} \mathrm{~K}^{-1}\right)$ & 753 \\
$T_{f}$ & fusion temperature $(\mathrm{K})$ & 1798 \\
$L$ & enthalpy of melting $\left(\mathrm{J} \mathrm{kg}^{-1}\right)$ & $2.77 \times 10^{-5}$ \\
$\lambda$ & thermal conductivity $\left(\mathrm{W} \mathrm{m}^{-1} \mathrm{~K}^{-1}\right)$ & 26 \\
$\lambda_{s}$ & solid thermal conductivity $\left(\mathrm{W} \mathrm{m}^{-1} \mathrm{~K}^{-1}\right)$ & 26 \\
\hline
\end{tabular}

welding wire are similar). The frequency $f$ of pulsed welding process is $113 \mathrm{~Hz}$ and the welding duration is $4 \mathrm{~s}$. During the different experiments, two parameters are set at three different levels:

- the wire feed speed can be set at 4, 6 and $8 \mathrm{~m} / \mathrm{min}$,

- and the initial temperature of the workpiece at 293, 573 and $873 \mathrm{~K}$.

Modification of wire speed changes welding energy and mass deposit rate. Welding energy influences heat transfer to melt the wire and the solid substrate: more the wire speed is important more the energy delivered by the process is important. The increase of the initial temperature eases the solid substrate melting: with the same energy, less energy is needed to melt the target with a higher $T_{0}$.

\subsection{Dimensionless procedure for spreading analysis of the macro-drop}

The behaviour of the macro-drop can be described with two kinds of parameters. The first one concerns the geometrical description of the macro-drop: base radius $R(\mathrm{~m})$, height $h(\mathrm{~m})$, volume $V\left(\mathrm{~m}^{3}\right)$ and contact angle $\theta$. The spatter is supposed negligible during liquid metal deposition and the volume $V$ of the macro-drop is assumed to be equal to the volume of liquid metal deposited. The second one is related to the spreading speed of the macro-drop and can be given by the average speed of displacement of the contact line $u\left(\mathrm{~m} \mathrm{~s}^{-1}\right)$. A spherical cap shape for the macro-drop is assumed so that if the deposited volume is known, the height $h$ and the contact angle $\theta$ can be computed. So the main purpose is to find the evolution of $R$ with respect to time.

The evolution of the spreading is expressed in term of the radius evolution of the macro-drop $R(t)$. To be adapted to various experimental conditions or process parameters, a dimensional analysis will be used with the set of major parameters that play a role on spreading. They can be divided into:

- Process parameters: the welding power $P=U I(\mathrm{~W})$ that takes also into account the contact tip to the workpiece distance, the wire feed speed, the wire diameter and the electrical resistivity. $U$ is the arc voltage and $I$ is the current delivered by the generator. $P$ is a good indicator for the energy delivered to the material. The frequency of impingement $f$ and the radius $r$ of droplets are parameters that can describe, when used together, the mass transfer into macro-drop. The mass input by time unit to the macro-drop can be expressed in function $v_{d}=f \frac{4}{3} \pi r^{3}$. The Weber number in relation with the droplets can be expressed as:

$$
W e=\frac{\rho u_{d}^{2} R}{\gamma}
$$

where $\rho$ and $\gamma$ are defined in Table 1 and $u_{d}$ is the velocity of the droplet at the impingement. The calculated Weber number is then $W e \leq 1$ indicating that inertial effects are lower than surface tension effects. It means that $u_{d}$ can be neglected in first investigation even if droplet velocity is multiplied by a factor 2 as it can be observed in other publications.

- Material parameters: $\rho_{s}, c_{s}$ and $\lambda_{s}$, the thermal properties of the solid target that can influence heat transfer, $\rho, c, \lambda$ the thermal properties of the liquid metal and $\mu$ the viscosity of the fluid. All the material parameters are kept constant with respect to temperature as a first hypothesis. With this hypothesis, the different ratio $\rho_{s} c_{s} / \rho c$ are fixed so only fluid properties are kept in the analysis.

- Considering the established macro-drop as global system, Reynolds number can be estimated at the contact line in order to describe the liquid spreading. The estimated value is $\operatorname{Re}=\frac{\rho u D}{\mu} \approx 47$ where $u=2.6 \mathrm{~mm} / \mathrm{s}$ is the average speed of the contact line and $D=15 \mathrm{~mm}$ the characteristic diameter of the macro-drop (see Fig. 2). The Reynolds number shows that the macrodrop spreading should not be directly affected by the fluid flows inside the macro-drop. The influence of viscosity in regard of surface tension can be estimated with the capillary number $\mathrm{Ca}$ :

$$
C a=\frac{\mu u}{\gamma}
$$

The capillary number is lower than $2 \times 10^{-5}$, underlining the minor effect of viscous forces in regard of surface tension forces. The viscosity $\mu$ seems not to be a significative parameter in the spreading, in a first approach. 
- Thermal parameters of the different domains that can influence heat transfer between the droplet and the macro-drop and between the macro-drop and the solid substrate. $T_{d}$ the temperature of the droplet and $T_{m}$ the macro-drop temperature are assumed to be at the melt temperature $T_{f}$ (no superheat assumption inside the macro-drop and the droplet). $T_{0}$ the temperature of the solid target and $L$ the latent heat of fusion are assumed to be constant all along the $4 \mathrm{~s}$ duration of the deposit. During the spreading, the latent heat of fusion is assumed to have no effect (high Stephan number). The thickness of the solid substrate used in experiments is large enough to neglect its influence in thermal boundary conditions and to assume conduction in a semi-infinite medium configuration. For wetting on thin plate, thickness has to be integrated in the set of parameters to describe spreading. As it is assumed that droplet and macro-drop are at $T_{f}$, the only important factor for heat transfer in the system is assumed to be the difference of the temperature between the liquid and the solid target $\Delta T=T_{f}-T_{0}$, combined probably with energy supplied by the process.

- Interface parameters: $\gamma$ the surface tension. Surface tension contributes to Marangoni effect that is due to the influence of $\frac{\mathrm{d} \gamma}{\mathrm{d} T}$ and that is known to play an important role in some application with high energy (high current) that creates high thermal gradient in the weld pool. In pulsed current, the short arc period is assumed to lead to an homogeneous temperature field inside the macrodrop. So Marangoni effect is not taken into account in the analysis. Parameters along the contact line is also not taken into account because they are too localized [7].

- Dynamic parameters: $g$ the gravity constant and Magnetic Field B. Once again, due to short period of the high current peak, the effects of electromagnetic force are neglected. The Bond number is defined as:

$$
B o=\frac{\rho g R^{2}}{\gamma}
$$

The Bond number $B o$ increases from low values at the initiation of the macro-drop to 2.5 at the end of experiments. The values underline a quite well balanced contribution of gravitational effects and capillary effects. There is a progressive higher influence of the gravity, in agreement with the increasing mass of the macrodrop due to the droplets deposit. To investigate the first stage of the spreading and in agreement with spherical cap shape observed during spreading, the effect of gravity will be neglected.

Finally, the evolution of the radius of the macro-drop is searched in function of:

$$
R(t)=f\left(P, v_{d}, r, \Delta T, \gamma, \rho, \lambda, c_{p}, t\right)
$$

These variables can be considered uncoupled. $\Delta T$ is only due to pre-heating and is not influenced by process parameters. The mass input by time unit $v_{d}$ and the radius $r$ of the droplet are uncoupled from the mean welding power. Droplet size can be controlled by peak current and time period of the peak for a same welding power, and a same mass input by time unit can be obtained both for different droplet sizes and different welding powers. Surface tension $\gamma$ mainly depends on gas composition. A dimensional analysis was used in order to identify the main significative variables. The Buckingham $\pi$-theorem $[14,15]$ was used to determine the appropriate dimensionless parameters containing the welding variables and material properties that affect the welding process. In particular, energy variables will be taken into account. If a system can be defined with $n$ variables that can be expressed in terms of $m$ fundamental units, namely for GMAW mass $[M]$, length $[L]$, time $[T]$, temperature $[\theta]$ and welding current $[I]$, the Buckingham $\pi$-theorem allows the system to be defined with the help of $n-m$ dimensionless groups $\Pi_{i}$ (the ' $\Pi$ ' label with numerical subscript is generally used to represent a dimensionless group of variables in dimensional analysis).

With the different assumptions, we can consider four fundamental units and ten variables for the description of the behaviour of the macro-drop system (see Table 2 in Appendix): the base radius $R$, the droplet radius $r$, mass input by time unit $v_{d}$, the time $t$, the welding power $P$, the surface tension $\gamma$, the temperature $\Delta T$, the thermal conductivity $\lambda$, the specific heat $c_{p}$ and the density $\rho$. The following six dimensionless groups are derived from these variables, on the base of the dimensional matrix presented Table 2:

$\Pi_{1}=\frac{R \rho c_{p}^{1.5} \sqrt{\Delta T}}{\lambda}$

$\Pi_{2}=\frac{r \rho c_{p}^{1.5} \sqrt{\Delta T}}{\lambda}$

$\Pi_{3}=\frac{v_{d} \rho^{2} c_{p}^{2.5} \sqrt{\Delta T}}{\lambda^{2}}$

$\Pi_{4}=\frac{t \rho c_{p}^{2} \Delta T}{\lambda}$

$\Pi_{5}=\frac{P \rho c_{p}^{1.5}}{\lambda^{2} \sqrt{\Delta T}}$

$\Pi_{6}=\frac{\gamma \sqrt{c_{p}}}{\lambda \sqrt{\Delta T}}$

The different parameters are related in a physical relationship: 
$\Phi\left(\Pi_{1}, \Pi_{2}, \Pi_{3}, \Pi_{4}, \Pi_{5}, \Pi_{6}\right)=\Pi$

where $\Pi$ can be a constant.

The dimensionless groups can be combined to yield meaningful dimensionless numbers such as the capillary number $\mathrm{Ca}$, the Weber number We, the Bond number $\mathrm{Bo}$, etc., as already discussed. They can also be combined in more indicative dimensionless parameters:

- the spread factor:

$$
\frac{\Pi_{1}}{\Pi_{2}}=\frac{R}{r}
$$

- and the dimensionless volume $V^{*}$ which corresponds to the ratio of the volume of liquid metal deposited and of the volume of a liquid droplet:

$$
V^{*}=\frac{3}{4 \pi} \frac{\Pi_{3} \Pi_{4}}{\Pi_{2}^{3}}
$$

This non dimensional number integrates the effect of time and takes into account mass conservation.

The dimensionless group $\Pi_{5}$ may be thought of as a dimensionless number that represents the energetic effects (due to welding power or/and initial temperature of the workpiece): it describes the energy supplied both by the welding power or preheating operations. $\Pi_{6}$ can be considered as a dimensionless number that traduces the effects of shielding gas composition on the surface tension of the metal. With this consideration, the Eq. (11) can be rewritten in a different form:

$\frac{R}{r}=\Psi\left(V^{*}, \Pi_{5}, \Pi_{2}, \Pi_{3}, \Pi_{6}\right)$

In the experimental tests $\Pi_{2}, \Pi_{3}, \Pi_{6}$ are not evolving during the experiments. So, the physical relationship can be written:

$\frac{R}{r}=v\left(V^{*}, \Pi_{5}\right) \chi\left(\Pi_{2}, \Pi_{3}, \Pi_{6}\right)$

It was assumed that the independence of parameters leads to a separation of function. Because during the experiments, the variables in the function $\chi$ are constant, the last term can be assumed to be constant in order to investigate our datas. All our data points obtained with temporal evolution are converted to a plot of $\frac{R}{r}$ in function of the two dimensionless number $V^{*}$ and $\Pi_{5}$ as shown in Fig. 4. With this representation, all the experimental points are gathered and it seems then possible to describe the evolution of the spreading of the macro-drop as:

$$
\frac{R(t)}{r}=g\left(\Pi_{5}, V^{*}\right)
$$

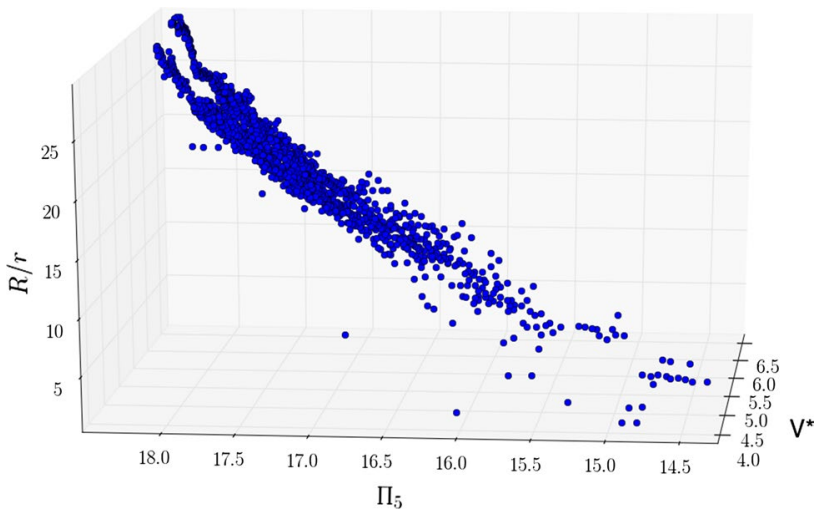

Fig. 4 Evolution of the spread factor $R / r$ in function of the two dimensionless parameters $V^{*}$ and $P_{5}$ that can vary in the different experiments. All the experimental data are gathered in a same surface

\section{Discussion on the macro-drop spreading}

The initial spreading of the macro-drop is quite huge in the beginning of the spreading due to the direct arc heating (average speed of displacement of the contact line $u=4.06 \mathrm{~mm} / \mathrm{s}$ ). After this initial rapid evolution, the spreading is more gradual with an average speed $u=1.23 \mathrm{~mm} / \mathrm{s}$ (Fig. 2). In this section, the anisothermal spreading is discussed in the light of non-dimensional number. First, classical dimensionless number are discussed, then the numbers of the previous section are used to investigate the spreading.

\subsection{Classical non dimensional numbers}

The spreading seems then to be driven by gravity effects and surface tension. The resistance to spreading can be analysed with the Ohnesorge number $Z$ that scales the force that resists the spreading:

$Z=\frac{\mu}{\sqrt{\rho \gamma R}}$

At high $Z$, the resistance is mainly viscous, and at low $Z$, it is mainly inertial [7]. In the initial spreading of the macro-drop, the Ohnesorge number $Z<0.012$ shows that the viscous forces can be mobilized, but this effect decreases in regards of inertial and surface tension forces in the gradual spreading period $\left(Z<10^{-3}\right)$. The ratio of the inertial forces to the surface tension forces is described by the Weber number We:

$W e=\frac{\rho R u^{2}}{\gamma}$

The low values of $W e$, in the range $10^{-3}-10^{-2}$, show the importance of surface tension effects on inertial 


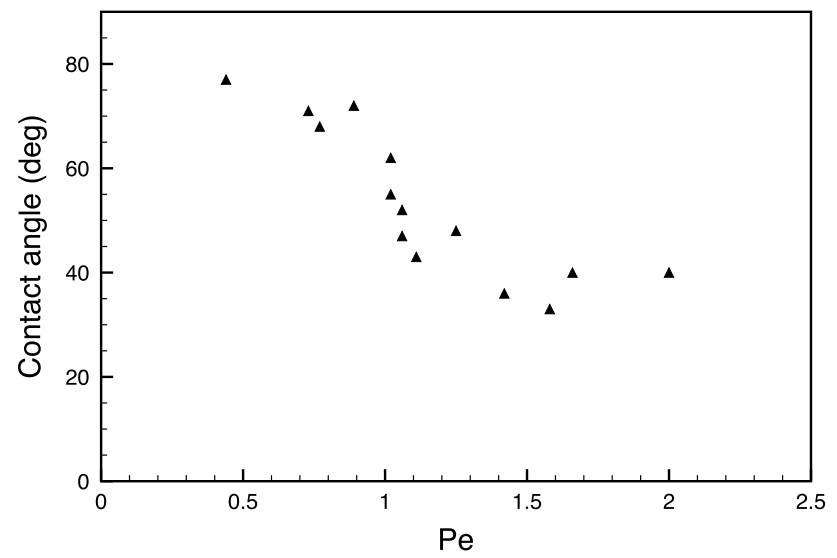

Fig. 5 Contact angle of the macro-drop vs Peclet number $P e$. The graph synthesises the data obtained for the different experiments. The values of contact angle and spreading speeds required for the calculation of $P e$ are the average values obtained before the welding stops

effects. It shows that the spreading of the macro-drop is governed by capillary force and limited by inertial forces on the contact line. The effects of droplet impact can be neglected (low momentum) and viscous forces are not significant.

The influence of the momentum diffusivity compared to the thermal diffusivity can be described by the Prandtl number $\operatorname{Pr}$ that only depends on the material used:

$\operatorname{Pr}=\frac{\mu c_{p}}{\lambda}$

In our experiments the relatively low value $\operatorname{Pr}=0.17$ shows that the heat diffuses very quickly; there is no high influence of velocity field on temperature field. The macroscopic Peclet number $P e$ can be interpreted as the ratio of heat transferred by bulk motion or advection of the base metal to the heat transferred in the base metal of the workpiece by conduction:

$P e=\frac{\rho c_{p} R u}{\lambda}$

The values of macroscopic $P e$ (see Fig. 5) show a quite well balanced contribution of convective and conductive transfers, with an higher contribution of convection. Figure 5 presents the evolution of the contact angle with Peclet number. We can note a decrease in contact angle and then an improved wetting of the macro-drop with the increasing values of $P e$.

\subsection{Non dimensional process parameters}

The way that the P-GMAW supplies heat and mass transfer to the workpiece also influences the spreading of the

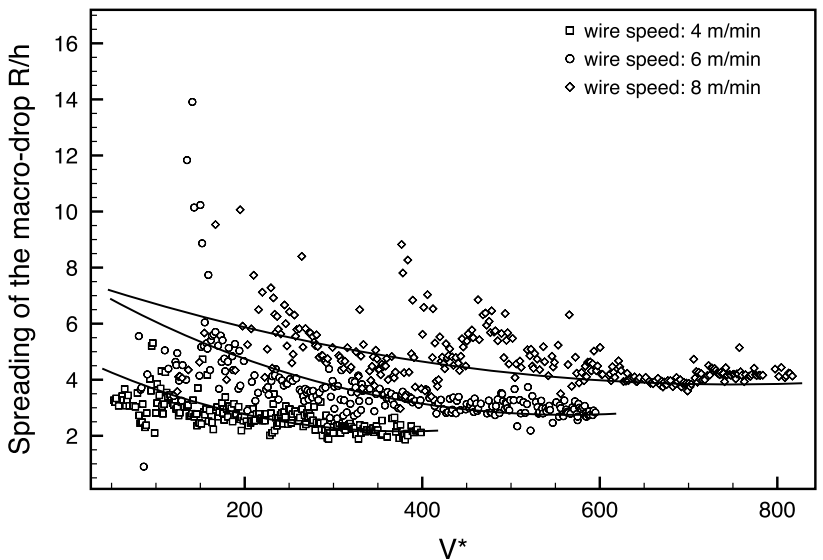

Fig. 6 Evolution of the shape factor $R / h$ of the macro-drop with the dimensionless volume for the different wire feed speed. The lines are drawn as a guide to the eyes

macro-drop. For example, the increase of wire feed speed increases the mass deposition rate but also the heat supplied to the workpiece. It is then possible to consider the evolution of the shape of the macro-drop $R / h$ with the dimensionless volume $V^{*}$ in relation with the wire feed speed. Figure 6 shows the influence of the wire feed speed on the spreading of the macro-drop; the spreading increases with the wire feed speed.

The increase of wire feed speed supplies higher quantity of liquid metal to the workpiece and also higher energy that contributes to facilitate the spreading of the macro-drop. The influence of energy on the spreading can be illustrated by taking into acçount the energetic dimensionless parameter $\Pi_{5}=\frac{P \rho c_{p}}{\lambda^{2} \sqrt{\Delta T}}$ (Fig. 7). The different plots seem to collapse in one plot; the global behaviour of the macro-drop seems to be governed by the mass/energy inputs.

Modifying the initial temperature $T_{0}$ of the workpiece is another possibility to supply heat to the system. Figure 8 presents the evolution of the base radius of the macro-drop for the different initial temperatures of the workpiece. The observed evolutions are nearly the same for the three different initial conditions of temperature.

On the large temperature range (200-800 K), the material properties change. The small difference can be attributed to the capacity of the material to diffuse heat. With increasing temperature, steel diffuses less heat.

The dimensionless parameter $\Pi_{5}$ is an interesting energetic indicator that traduces the different ways that heat can influence the macro-drop system. It takes into account the global direct energy supplied to the macrodrop system by the arc welding process through the welding power quantity $P$; but it also takes into account the contribution of the initial temperature condition of the solid workpiece through the difference of temperatures 


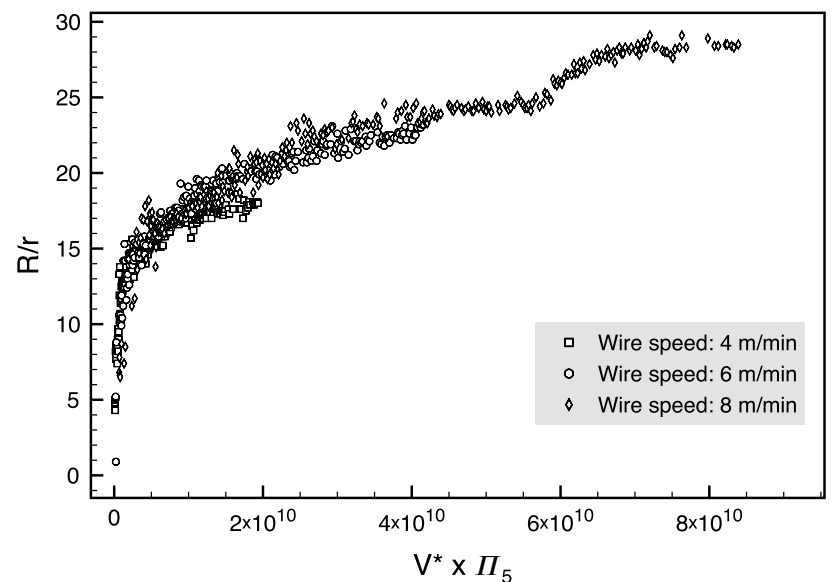

Fig. 7 Evolution of the spread factor $R / r$ of the macro-drop in relation with mass transfer and energy effects due to the wire feed speed

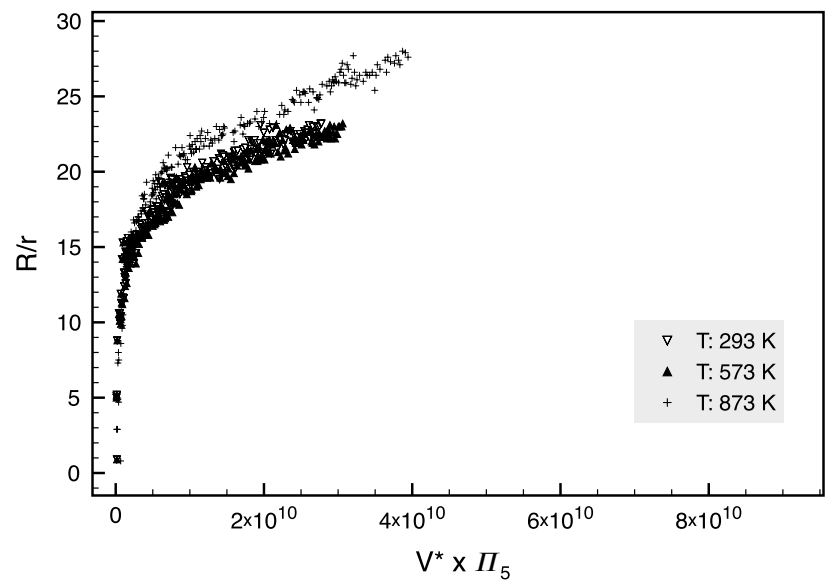

Fig. 8 Evolution of the spread factor $R / r$ of the macro-drop in relation with mass transfer and energy effects due to the pre-heating of the workpiece

$\Delta T$ and its ability of heat sink with the thermal conductivity $\lambda$.

The spreading of a liquid drop impacting a solid surface is classically described as a power function $[2,5,9$, $16,17]$. Figure 9 presents the evolution of the spread factor $R / r$ for all the different conditions used in the experiments as Eq. (16) proposed. For the whole set of data, all the experimental points are gathered on a same curve based on power functions of $V^{*}$ and $\Pi_{5}$. In this non-isothermal configuration of a regularly fed macro-drop of liquid metal, the spreading can also be described by an empirical power function taking into account heat and mass transfers:

$\frac{R}{r}=a V^{* n} \Pi_{5}^{m}$

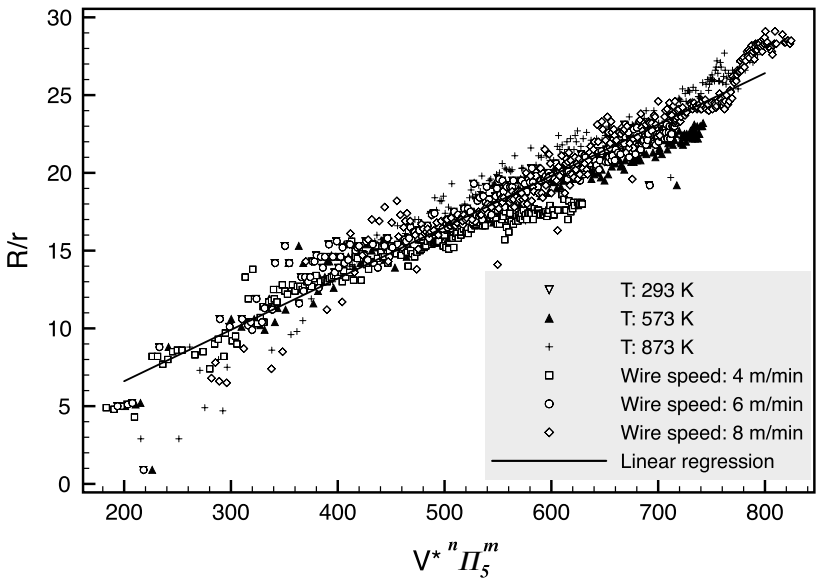

Fig. 9 Evolution of the spread factor $R / r$ of the macro-drop in relation with mass transfer and energy effects. Comparison for several possibilities in heat transfer

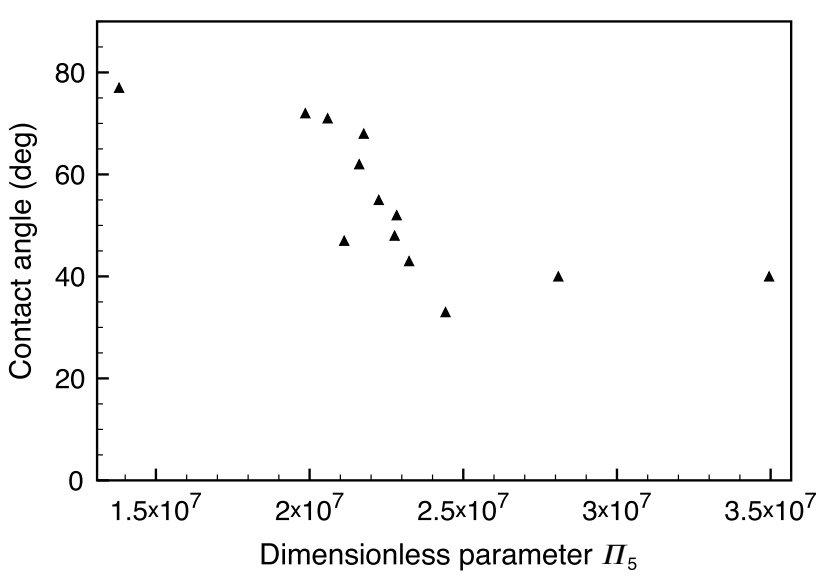

Fig. 10 Contact angle of the macro-drop vs dimensionless parameter $\Pi_{5}$. The graph synthesizes the data obtained for the different types of experiments. The values of contact angle are the average values obtained before the welding stops

where $a=0.033, n=0.003541$ and $m=0.3627$. Whatever the experimental conditions, it is then possible to evaluate the spreading of the macro-drop of liquid metal with a unique relation giving the evolution of the base radius of the macro-drop as a power function of volume and energetic parameter.

The heat transferred to the system contributes to improve the wetting of the macro-drop as underlined on Fig. 10. An important decrease in the average values of contact angle can be noted when the dimensionless parameter $\Pi_{5}$ increases. Independently of the way that heat is transferred to the workpiece, the heat input facilitates the wetting of liquid metal on solid base metal and the spreading of the macro-drop. 


\section{Conclusion}

The deposition of liquid metal droplets on a solid metal substrate is a complex mechanism that involves several physical phenomena. In this study, mass and heat transfers are supplied by P-GMAW process to a solid metal workpiece, in static configuration. During the initiation of the deposit, the direct arc heating creates a small fusion zone on the solid target and we can observe first a rapid spreading of the macro-drop. In a second phase, the mass transfer allows a regular growing of the macro-drop and a regular spreading of the contact line. This spreading can be influenced and modified by process parameters modifications, such as welding speed or initial temperature of the solid target. A dimensional analysis was used to identify the main physical phenomena involved in the spreading and wetting of the macro-drop in relation with the process parameters. The different experiments and observations show that the global behaviour of the macro-drop is governed on by capillary effects and gravity that give the global shape of the liquid bulk. It also appears that the heat input is an important point in the behaviour of liquid metal bulk by facilitating the wetting and the spreading of the macro-drop. In non-isothermal conditions, in the very severe conditions of gas metal arc welding, a new relation was proposed to describe the spreading of the macro-drop of liquid metal with a unique expression giving the evolution of the base radius of the macro-drop as a power function of volume and energetic parameter. This relation describes the evolution of liquid metal drop on a solid substrate in fuction of mass and heat inputs. In a practical point of view, such a relation could be useful to estimate the global shape and spreading of liquid metal deposits on the base of the process parameters in industrial process such as metal arc welding or cladding for example. Further developments of this approach are also in progress in order to study complex problems of instabilities in liquid metal deposition and problems of contact line behaviour in non-isothermal configurations.

\section{Appendix}

See Table 2.
Table 2 Dimensional matrix and dimensionless groups

\begin{tabular}{|c|c|c|c|c|c|c|c|c|c|c|}
\hline & \multirow{2}{*}{$\begin{array}{l}\text { Output } \\
R\end{array}$} & \multicolumn{9}{|c|}{ Input } \\
\hline & & $r$ & $v_{\mathrm{d}}$ & $t$ & $P$ & $\gamma$ & $\Delta T$ & $c_{p}$ & $\lambda$ & $\rho$ \\
\hline Length (L) & 1 & 1 & 3 & 0 & 2 & 0 & 0 & 2 & 1 & -3 \\
\hline Mass (M) & 0 & 0 & 0 & 0 & 1 & 1 & 0 & 0 & 1 & 1 \\
\hline Time (T) & 0 & 0 & -1 & 1 & -3 & -2 & 0 & -2 & -3 & 0 \\
\hline Temperature $(\theta)$ & 0 & 0 & 0 & 0 & 0 & 0 & 1 & -1 & -1 & 0 \\
\hline$P_{1}$ & 1 & 0 & 0 & 0 & 0 & 0 & 0.5 & 1.5 & -1 & 1 \\
\hline$P_{2}$ & 0 & 1 & 0 & 0 & 0 & 0 & 0.5 & 1.5 & -1 & 1 \\
\hline$P_{3}$ & 0 & 0 & 1 & 0 & 0 & 0 & 1.5 & 4.5 & -3 & 3 \\
\hline$P_{4}$ & 0 & 0 & 0 & 1 & 0 & 0 & -0.5 & -0.5 & 0 & 0 \\
\hline$P_{5}$ & 0 & 0 & 0 & 0 & 1 & 0 & -0.5 & 1.5 & -2 & 1 \\
\hline$P_{6}$ & 0 & 0 & 0 & 0 & 0 & 1 & -0.5 & 1.5 & -2 & 1 \\
\hline
\end{tabular}




\section{References}

1. Gao F, Sonin AA (1994) Precise deposition of molten microdrops: the physics of digital microfabrication. Proc R Soc A 444(1922):533-554

2. De Gennes PG (1985) Wetting: statics and dynamics. Rev Mod Phys 57(3):827-863

3. Li R, Ashgriz N, Chandra S, Andrews JR (2007) Solidification contact angles of molten droplets deposited on solid surfaces. J Mater Sci 42:9511-9523

4. Lim YC, Farson DF, Cho MH, Cho JH (2009) Stationary GMAW-P weld metal deposit spreading. Sci Technol Weld Join 14(17):626-635

5. Bonn D, Eggers J, Indekeu J, Meunier J, Rolley E (2009) Wetting and spreading. Rev Mod Phys 81:739-805

6. Snoeijer JH, Andreotti B (2013) Moving contact lines: scales, regimes and dynamical transitions. Annu Rev Fluid Mech 45:269-292

7. Schiaffino S, Sonin AA (1997) Molten droplet deposition and solidification at low Weber numbers. Phys Fluids 9(11):3172-3187

8. Chapuis J, Romero E, Soulié F, Bordreuil C, Fras G (2013) Behaviour of spreading molten metal drops deposited by fusion. Exp Therm Fluid Sci 48:29-36
9. Biance AL, Clanet C, Quéré D (2004) First steps in the spreading of a liquid droplet. Phys Rev E 69(016301):1-4

10. Arora A, Roy GG, DebRoy T (2009) Unusual wavy weld pool boundary from dimensional analysis. Scr Mater 60:68-71

11. van Elsen A, Al-Bender F, Kruth JP (2008) Application of dimensional analysis to selective laser melting. Rapid Prototyp J 14(1):15-22

12. Nguyen TC, Weckman DC, Johnson DA (2007) Predicting onset of high speed gas metal arc weld bead defects using dimensional analysis techniques. Sci Technol Weld Join 12(7):634-648

13. Romero E, Chapuis J, Bordreuil C, Soulié F, Fras G (2013) Image Processing and geometrical analysis for profile detection during P-Gas Metal Arc Welding. Proc Inst Mech Eng B J Eng Manuf 227:396-406

14. Barenblatt GI (2003) Scaling. Cambridge University Press, Cambridge

15. Bridgman PW (1922) Dimensional analysis. Yale University Press, New Haven

16. Tanner LH (1979) The spreading of silicone oil drops on horizontal surfaces. J Phys D Appl Phys 12:1473-1484

17. Eddi A, Winkels KG, Snoeijer JH (2013) Short time dynamics of viscous drop spreading. Phys Fluids 25(013102):1-10 